PDFlib PLOP: PDF Linearization, Optimization, Protection

Page inserted by evaluation version www.pdflib.com - sales@pdflib.com 


\title{
Relative Equilibria for General Gravity Fields in the Sphere-Restricted Full Two-Body Problem
}

\author{
D.J. SCHEERES
}

Aerospace Engineering Department, The University of Michigan,

Ann Arbor, Michigan, USA

\begin{abstract}
Equilibrium conditions for a mutually attracting general mass distribution and point mass are stated. The equilibrium conditions can be reduced to six equations in six unknowns, plus the existence of integrals of motion consisting of the total angular momentum and energy of the system. The equilibrium conditions are further reduced to two independent equations, and their theoretical properties are studied. We state a set of necessary and sufficient conditions for an equilibrium that is well suited to the computation of certain classes of equilibria. These equations are solved for nonsymmetric gravity fields of interest, using a real asteroid shape model for the general gravity fields. The stability of the resulting equilibria are also noted.
\end{abstract}

KEYWORDS: relative equilibria; general gravity fields; sphere-restricted full two-body problem

\section{INTRODUCTION}

The study of relative equilibria between massive bodies has received much interest over the years because many solar system bodies have been found to lie in or close to relative equilibria. Examples include the Moon relative to the Earth, the Galilean satellites relative to Jupiter, and more recently asteroid binary secondaries with respect to their primaries. The study of this problem requires one to solve for, and analyze, the mutual gravitational attractions and torques between two nonspherical bodies. Usually, one of the bodies (generally the larger) is not in a synchronous rotation state, giving rise to periodic perturbations in the system. The study of these general systems in a quasiequilibrium, where one of the bodies is synchronous but the other is not, is quite complex and is taxing to current analytic and numeric methods. A detailed discussion of this problem is given elsewhere. ${ }^{1-3}$

In this paper we do not consider this most general problem, one we have termed the full two-body problem ${ }^{4}$ but instead study a one-stage simplification of it, the sphere-restricted full two-body problem (SRF2BP), which arises when one of the bodies is a sphere (i.e., a point mass). Even for such a system, however, there are only limited results. To motivate future studies of the more complete system, we perform a study of the SRF2BP that goes beyond previous work in that a set of practical

Address for correspondence: D.J. Scheeres, Aerospace Engineering Department, The University of Michigan, Ann Arbor, MI 48109, USA.

scheeres@umich.edu

Ann. N.Y. Acad. Sci. 1065: 375-390 (2005). (C2005 New York Academy of Sciences. doi: 10.1196/annals.1370.028 
algorithms for its solution are found and analyzed. One key assumption of nearly all previous studies, however, is that the gravity field of the non-spherical body can be approximated with its second degree and order gravity field. Exceptions that exist generally only describe the general process of determining the equilibrium configurations of a more general system without specific results for specific bodies. ${ }^{2}$ In this paper we address this limitation by deriving conditions that can be used to compute relative equilibrium in the SRF2BP for general gravity fields. This is motivated by understanding and studying the dynamics of binary asteroid systems during their formation process.

We suspect that most relative equilibria between two general asteroids will be when the distance is small, and only become stable as their distance increases, consistent with ellipsoidal models. ${ }^{5}$ These questions are of interest for understanding the dynamics and initial evolution of binary asteroid systems after their formation. As a result of our study, we also shed light on previous results derived under the seconddegree and order gravity field assumption.

In this paper, we specifically derive equilibrium conditions for a mutually attracting general mass distribution and point mass. The full dimensionality of this system has nine degrees of freedom, and hence, 18 separate differential equations. The equilibrium conditions can be easily reduced to six equations in six unknowns, plus the existence of four integrals of motion consisting of the total angular momentum and energy of the system. We further reduce the equilibrium conditions to two independent equations and study their theoretical properties. We solve these equations for a non-symmetric gravity field of interest and determine their spectral and energetic stability.

There has been much previous work in this area. ${ }^{1-3,5}$ Our contribution is to perform an in-depth analysis of the equilibrium equations themselves and reduce them to a set of two equations. We find three different forms of these equations, a set that defines the necessary conditions for equilibrium, a set that defines sufficient conditions for equilibrium, and a set that defines necessary and sufficient conditions. In the current contribution we only present the necessary and sufficient conditions, leaving the others for a more comprehensive article. Using these equations we study theoretical properties of these solutions and compute relative equilibria for a general mass distribution.

\section{FUNDAMENTALS}

We omit a complete derivation of the equations of motion and only state them in an appropriate form. For in-depth derivations of this problem, see elsewhere. $1,2,5,6$ In the following, we are concerned with stating the equations of motion for a general mass distribution and a sphere. These equations can be reduced to the relative motion between the bodies and the rotational dynamics of the general body. In fact, the equations of motion for the spin vector of the body can be decoupled from its orientation, which is then solved after the fact. Our problem is analyzed most simply if we transform to a coordinate frame fixed to the rotating, general body. The main advantage of stating the equations of motion in this frame is that this removes the attitude of the body from consideration. 


\section{Equations of Motion in a Rotating Frame}

The general equations of motion for our system are stated as follows:

$$
\begin{gathered}
\ddot{\mathbf{R}}+2 \boldsymbol{\Omega} \times \dot{\mathbf{R}}+\dot{\boldsymbol{\Omega}} \times \mathbf{R}+\boldsymbol{\Omega} \times(\boldsymbol{\Omega} \times \mathbf{R})=G\left(M_{c}+M_{s}\right) \frac{\partial U}{\partial \mathbf{R}} \\
\mathbf{I} \cdot \dot{\boldsymbol{\Omega}}+\boldsymbol{\Omega} \times \mathbf{I} \times \boldsymbol{\Omega}=-G M_{c} M_{s} \mathbf{R} \times \frac{\partial U}{\partial \mathbf{R}},
\end{gathered}
$$

where $\mathbf{R}$ is the relative position vector between the bodies, $\boldsymbol{\Omega}$ is the angular velocity vector, $M_{c}$ is the mass of the non-spherical body, $M_{s}$ is the mass of the sphere, and $G$ is the gravitational constant. The unit mass gravity potential is $U$ and the inertia dyad is $\mathbf{I}$, defined by

$$
\begin{gathered}
U(\mathbf{R})=\frac{1}{M_{c}} \int_{\beta_{c}} \frac{d m_{c}(\boldsymbol{\rho})}{|\mathbf{R}+\boldsymbol{\rho}|} \\
\mathbf{I}=-\int_{\beta_{c}} \tilde{\boldsymbol{\rho}} \cdot \tilde{\boldsymbol{\rho}} d m(\boldsymbol{\rho}),
\end{gathered}
$$

where $\boldsymbol{\rho}$ is the position vector of the mass element $d m$ in the general body, $\beta_{c}$ represents the mass distribution, and $\tilde{\boldsymbol{\rho}}$ denotes the cross-product dyad. In general, given a vector $\boldsymbol{\rho}=\left[\rho_{x}, \rho_{y}, \rho_{z}\right]$ we have

$$
\tilde{\boldsymbol{\rho}}=\left[\begin{array}{ccc}
0 & -\rho_{z} & \rho_{y} \\
\rho_{z} & 0 & -\rho_{x} \\
-\rho_{y} & \rho_{x} & 0
\end{array}\right] .
$$

The relative vector $\mathbf{R}$ can be expressed in terms of the two inertial centers of mass of the body

$$
\begin{aligned}
\mathbf{R} & =\mathbf{A}^{T} \cdot \mathbf{r}_{I} \\
& =\mathbf{A}^{T} \cdot\left(\mathbf{r}_{s}-\mathbf{r}_{c}\right)
\end{aligned}
$$

where $\mathbf{A}$ is the attitude matrix of the general body (i.e., the transformation matrix that takes the general body frame to the inertial frame), $\mathbf{A}^{T}$ is the transformation matrix that goes from the inertial frame to the general body frame, $\mathbf{r}_{I}$ is the relative vector between the bodies in inertial space, and $\mathbf{r}_{i}$ is the inertial position vector of the $i$ th body.

The evolution of the attitude matrix follows a simple equation,

$$
\dot{\mathbf{A}}=\mathbf{A} \times \mathbf{\Omega} .
$$

However, we find it convenient to use the more fundamental definition of attitude from the axis-angle coordinates, which describe the attitude of a rigid body by a rotation of angle $\phi$ about an axis of $\hat{\mathbf{a}}$, where the hat notation denotes a unit vector. In these coordinates, the attitude matrix can be specified as

$$
\mathbf{A}=\cos \phi \mathbf{U}+(1-\cos \phi) \hat{\mathbf{a}} \hat{\mathbf{a}}-\phi \tilde{\hat{\mathbf{a}}},
$$

where $\mathbf{U}$ is a unit dyad, that is, $\mathbf{U} \cdot \mathbf{a}=\mathbf{a} \cdot \mathbf{U}=\mathbf{a}$ for any vector $\mathbf{a}$. In a body-fixed frame, the axis-angle coordinates follow their own equations of motion, 


$$
\begin{gathered}
\dot{\hat{\mathbf{a}}}=\frac{1}{2}\left[\tilde{\hat{\mathbf{a}}}-\cot \left(\frac{\phi}{2}\right) \tilde{\hat{\mathbf{a}}} \cdot \tilde{\hat{\mathbf{a}}}\right] \cdot \mathbf{\Omega} \\
\dot{\phi}=\dot{\hat{\mathbf{a}}} \cdot \boldsymbol{\Omega} .
\end{gathered}
$$

This system, as stated above, has four integrals of motion defined for it, the total energy and the total angular momentum. Specifically, these are

$$
\begin{gathered}
E=\frac{1}{2} \frac{M_{s} M_{c}}{M_{s}+M_{c}}(\dot{\mathbf{R}}+\boldsymbol{\Omega} \times \mathbf{R}) \cdot(\dot{\mathbf{R}}+\boldsymbol{\Omega} \times \mathbf{R})+\frac{1}{2} \boldsymbol{\Omega} \cdot \mathbf{I} \cdot \boldsymbol{\Omega}-G M_{s} M_{c} U(\mathbf{R}) \\
\mathbf{K}=\mathbf{A} \cdot\left[\mathbf{I} \cdot \boldsymbol{\Omega}+\frac{M_{c} M_{s}}{M_{c}+M_{s}}\{\mathbf{R} \times \dot{\mathbf{R}}+\mathbf{R} \times(\boldsymbol{\Omega} \times \mathbf{R})\}\right]
\end{gathered}
$$

\section{Equations of Motion in a Normalized Frame}

The equations can be simplified by introducing three normalizations: a length scale, a time scale, and normalizing the inertia $\mathbf{I}$ by the total mass of the nonspherical body, $M_{c}$, as well as the length scale squared. For the length scale we take an arbitrary radius (often the maximum radius of the distributed body), denoted by $\alpha$. To define the time scale we take the mean motion of the total system at this radius,

$$
n=\sqrt{\frac{G\left(M_{c}+M_{s}\right)}{\alpha^{3}}},
$$

and define the normalized position vectors, $\mathbf{r}=\mathbf{R} / \alpha$, and the normalized rotational velocity vectors, $\boldsymbol{\omega}=\boldsymbol{\Omega} / n$. Finally, we rescale the inertia matrix by dividing it by $M_{c} \alpha^{2}$. In the following, we define the new time derivatives by $(\cdot)^{\prime}$. This yields the following set of equations:

$$
\begin{gathered}
\mathbf{r}^{\prime \prime}+2 \boldsymbol{\omega} \times \mathbf{r}^{\prime}+\boldsymbol{\omega}^{\prime} \times \mathbf{r}+\boldsymbol{\omega} \times(\boldsymbol{\omega} \times \mathbf{r})=\frac{\partial \mathcal{U}}{\partial \mathbf{r}} \\
\mathcal{I} \cdot \boldsymbol{\omega}^{\prime}+\boldsymbol{\omega} \times \mathcal{I} \cdot \boldsymbol{\omega}=-v \mathbf{r} \times \frac{\partial \mathcal{U}}{\partial \mathbf{r}},
\end{gathered}
$$

where the normalized inertia matrix and gravitational force potential are

$$
\begin{gathered}
\mathcal{I}=-\frac{1}{M_{c} \alpha} \int_{\beta_{c}} \tilde{\boldsymbol{\rho}} \cdot \tilde{\boldsymbol{\rho}} d m \\
\mathcal{U}=\frac{\alpha}{M_{c}} \int_{\beta_{c}} \frac{d m}{|\mathbf{r}+\boldsymbol{\rho}|},
\end{gathered}
$$

respectively.

The equations for the axis-angle variables are

$$
\begin{gathered}
\hat{\mathbf{a}}^{\prime}=\frac{1}{2}\left[\tilde{\hat{\mathbf{a}}}-\cot \left(\frac{\phi}{2}\right) \tilde{\hat{\mathbf{a}}} \cdot \tilde{\hat{\mathbf{a}}}\right] \cdot \boldsymbol{\omega} \\
\phi^{\prime}=\hat{\mathbf{a}} \cdot \boldsymbol{\omega},
\end{gathered}
$$

and the transformed integrals of motion are

$$
\mathbf{k}=\mathbf{A} \cdot\left[\mathcal{I} \cdot \boldsymbol{\omega}+v \mathbf{r} \times\left(\mathbf{r}^{\prime}+\boldsymbol{\omega} \times \mathbf{r}\right)\right]
$$




$$
\mathcal{E}=\frac{v}{2}\left(\mathbf{r}^{\prime}+\boldsymbol{\omega} \times \mathbf{r}\right) \cdot\left(\mathbf{r}^{\prime}+\boldsymbol{\omega} \times \mathbf{r}\right)+\frac{1}{2} \boldsymbol{\omega} \cdot \mathcal{I} \cdot \boldsymbol{\omega}-v \mathcal{U}
$$

One of the free parameters of the system is

$$
v=\frac{M_{s}}{M_{s}+M_{c}},
$$

which is the same parameter as is found in the restricted three body problem. The case $v \rightarrow 0$ corresponds to the motion of a material point in the gravity field of the distributed body, with main application to orbital dynamics about an asteroid. In this case, we see that the energy and angular momentum integrals are dominated by the rotational dynamics of the distributed body, and that the contribution of the motion of the spherical body decouples from these integrals. The case $v \rightarrow 1$ corresponds to the motion of a massless distributed body about a point-mass, with an application to a large satellite in orbit about a planet. It is important to note that the angular momentum and energy integrals still apply to this problem. There is no singularity as $M_{c} \rightarrow 0$ since the inertia dyad and gravity field of the distributed body are defined relative to the geometry alone. This indicates that analysis of gravity gradient satellites should not neglect translational motion, because it is coupled at this fundamental level to the rotational motion. To simplify our later computations of equilibria for some real asteroid models we leave the length unit unchanged (i.e., $\alpha=1$ ) but apply the time normalization to eliminate the total mass from the problem.

\section{Energy at a Constant Angular Momentum}

Of specific interest later in this paper is the form of the energy when a constant angular momentum vector is imposed. We first note that the angular momentum integral can be rewritten in the form

$$
\mathbf{k}=\mathbf{A} \cdot\left\{[\mathcal{L}-v \tilde{\mathbf{r}} \cdot \tilde{\mathbf{r}}] \cdot \boldsymbol{\omega}+v \tilde{\mathbf{r}} \cdot \mathbf{r}^{\prime}\right\}
$$

and that $\boldsymbol{\omega}$ can be solved for to obtain

$$
\begin{gathered}
\boldsymbol{\omega}=\mathcal{J}^{-1} \cdot\left[\mathbf{A}^{T} \cdot \mathbf{k}-v \tilde{\mathbf{r}} \cdot \mathbf{r}^{\prime}\right] \\
\mathcal{J}=\mathcal{I}-v \tilde{\mathbf{r}} \cdot \tilde{\mathbf{r}} .
\end{gathered}
$$

We note that the dyad $\mathcal{J}$ is never singular (assuming that $\mathcal{I}$ is not singular) and will play an important role later in our analysis. Physically, $\mathcal{J}$ is just the normalized inertia tensor of the entire binary system relative to the non-spherical body center of mass, also known as the "locked inertia" from Marsden and Simo.

Taking this result, we can eliminate $\boldsymbol{\omega}$ from explicitly appearing in the energy equation to find

$$
\mathcal{E}=\frac{v}{2} \mathbf{r}^{\prime} \cdot\left[\mathbf{U}+\tilde{\mathbf{r}} \cdot \mathcal{J}^{-1} \cdot \tilde{\mathbf{r}}\right] \cdot \mathbf{r}^{\prime}+\frac{1}{2} \mathbf{k} \cdot \mathbf{A} \cdot \mathcal{J}^{-1} \cdot \mathbf{A}^{T} \cdot \mathbf{k}-v \mathcal{U}
$$

Although the explicit dependence of $\boldsymbol{\omega}$ is removed, we note that this occurs at the cost of introducing the attitude matrix $\mathbf{A}$ into the energy. A more useful observation is that the magnitude of the angular momentum is only a function of the "internal variables" $\mathbf{r}, \mathbf{r}^{\prime}$, and $\boldsymbol{\omega}$. On the other hand, the unit vector along the angular momentum vector, $\mathbf{k}$, is a function of $\mathbf{A}$ as well. Arising from this fact, we can only eliminate one of the internal variables using the angular momentum. 


\section{EQUILIBRIUM CONDITIONS}

In the following, starting from the equations of motion, we derive a number of different versions of the equilibrium conditions. Our main focus is to reduce the conditions to the minimum number possible, which is two. Such a reduction makes it feasible to solve for the equilibrium conditions numerically for a specified gravity field. We find, however, that different versions of the conditions are more suited to finding particular types of equilibria.

\section{Fundamental Equations}

In the above form, the analysis of equilibrium solutions is relatively easy. The conditions for equilibrium (i.e., that all time derivatives be zero) are

$$
\begin{gathered}
\hat{\mathbf{a}}=\hat{\boldsymbol{\omega}} \\
\tilde{\boldsymbol{\omega}} \cdot \tilde{\boldsymbol{\omega}} \cdot \mathbf{r}=\frac{\partial \mathcal{U}}{\partial \mathbf{r}} \\
\tilde{\boldsymbol{\omega}} \cdot \mathcal{I} \cdot \boldsymbol{\omega}+v \tilde{\mathbf{r}} \cdot \frac{\partial \mathcal{U}}{\partial \mathbf{r}}=0 .
\end{gathered}
$$

The first equation just places constraints on the axis of rotation. Equations (28) and (29) constitute a set of six equations for six unknowns, the relative position $\mathbf{r}$ and the angular velocity $\boldsymbol{\omega}$. The solution of these equations is non-trivial, especially for a non-symmetric mass distribution for the gravitational potential. It is important to note that for $v=0$ the solution for Equation (29) reduces to the classic rigid body rotation results, equilibria are rotations about the principal axes of inertia. Given this, solutions to Equation (28) are then equilibrium points of a particle in the field of the uniformly rotating body, a subject studied previously for irregular mass distributions. $^{7}$

By definition, a system in equilibrium will have a constant uniform rotation vector $\boldsymbol{\omega}$ defined by its rotation rate $\omega=|\boldsymbol{\omega}|$ and its unit vector $\hat{\boldsymbol{\omega}}$, and thus, the transformation matrix A will have the specific form

$$
\mathbf{A}=\cos (\omega t) \mathbf{I}+(1-\cos (\omega t)) \hat{\boldsymbol{\omega}} \hat{\boldsymbol{\omega}}+\tilde{\hat{\boldsymbol{\omega}}} \sin (\omega t) \text {. }
$$

The presence of a uniformly increasing angle, $\omega t$, signifies that this is a relative equilibrium. We also note that the spin axis is a unity eigenvector of this transformation matrix, or $\mathbf{A} \cdot \boldsymbol{\omega}=\boldsymbol{\omega}$.

At an equilibrium the integrals of motion reduce to

$$
\begin{gathered}
\mathbf{k}=\mathbf{A} \cdot \mathcal{J} \cdot \boldsymbol{\omega} \\
\mathcal{E}=\frac{1}{2} \boldsymbol{\omega} \cdot \mathcal{J} \cdot \boldsymbol{\omega}-v \mathcal{U} .
\end{gathered}
$$

\section{Implications of the Equilibria}

There are a number of fundamental observations that we can make about the relative geometry of the system and conditions on the system when it satisfies the equilibrium solutions. These observations are not new; many were noted previously. 1,2 We restate them here since we can establish these results in a very simple manner, 
and to allow us to use them in our later discussion concerning the reduction of the equilibrium conditions.

Proposition 1: The spin axis is perpendicular to the gravitational acceleration.

Consider (28). Taking the dot product of $\boldsymbol{\omega}$ and $\mathcal{U}_{\mathbf{r}}$ we find

$$
\boldsymbol{\omega} \cdot \mathcal{U}_{\mathbf{r}}=(\boldsymbol{\omega} \cdot \mathbf{r}) \omega^{2}-\omega^{2}(\boldsymbol{\omega} \cdot \mathbf{r})=0 \text {. }
$$

Proposition 2: The spin axis is an eigenvector of [ $\mathcal{I}-v \mathbf{r r}]$.

Insert (28) for the gravitational acceleration into (29) to find

$$
\boldsymbol{\omega} \times \mathcal{I} \cdot \boldsymbol{\omega}+v(\mathbf{r} \cdot \boldsymbol{\omega}) \mathbf{r} \times \boldsymbol{\omega}=0,
$$

which can be rewritten as

$$
\boldsymbol{\omega} \times[\mathcal{I}-v \mathbf{r r}] \cdot \boldsymbol{\omega}=0
$$

where $\mathbf{r r}$ is a dyad. Assuming that $|\boldsymbol{\omega}| \neq 0$, this is true if and only if $[\mathcal{I}-v \mathbf{r r}] \cdot \boldsymbol{\omega}$ is parallel to $\omega$, or

$$
[\mathcal{I}-v \mathbf{r r}] \cdot \boldsymbol{\omega}=\sigma \boldsymbol{\omega}
$$

where $\sigma$ is an eigenvalue of the dyadic $\mathcal{I}-\operatorname{vrr}$ and $\boldsymbol{\omega}$ is its eigenvector.

Corollary 3: The spin axis is an eigenvector of $\mathcal{J}=[\mathcal{I}-v \tilde{\mathbf{r}} \cdot \tilde{\mathbf{r}}]$.

Note that $-\tilde{\mathbf{r}} \cdot \tilde{\mathbf{r}}=r^{2} \mathbf{U}-\mathbf{r r}$. Thus, this is rewritten as $[\mathcal{I}-v \mathbf{r r}]+v r^{2} \mathbf{U}$. From Proposition 2 the spin axis is an eigenvector of the first term, and every vector is an eigenvector of the identity dyad, completing the proof.

Corollary 4: The angular momentum vector is parallel to the spin axis.

Note that the angular momentum equation can be written: $\mathbf{k}=\mathbf{A} \cdot[\mathcal{I}-\tilde{\mathbf{r}} \cdot \tilde{\mathbf{r}}] \cdot \boldsymbol{\omega}$. The spin vector is an eigenvector of both dyads, and thus, $\mathbf{k}$ must be parallel to it, and will be an eigenvector to these dyads as well.

Corollary 5: The eigenvalue of the $\mathcal{J}$ dyad along the angular velocity is the ratio of the angular momentum magnitude over the angular velocity magnitude.

Note that $k=|\mathbf{k}| \hat{\mathbf{k}}=\mathbf{A} \cdot \mathcal{J} \cdot \boldsymbol{\omega}=\lambda \mathbf{A} \cdot \boldsymbol{\omega}=\lambda \boldsymbol{\omega}=\lambda \omega \hat{\boldsymbol{\omega}}$. However, $\hat{\mathbf{k}}=\hat{\boldsymbol{\omega}}$ from Corollary 4 , leading to $\lambda=|\mathbf{k}| / \omega$.

Proposition 6: The relative position vector lies in the plane defined by the gravitational acceleration and the spin axis.

This can be directly shown by rearranging Equation (28) into the form

$$
\mathbf{r}=\frac{1}{\omega^{2}}(\boldsymbol{\omega} \cdot \mathbf{r}) \boldsymbol{\omega}-\frac{1}{\omega^{2}} \mathcal{U}_{\mathbf{r}}
$$

Proposition 7: Stationary values of the energy are a necessary condition for equilibrium.

To establish this, assume that the system is in relative equilibrium and then show that the energy is at a stationary point. When we take the variation of energy, we note that the angular momentum must be held constant. Starting from (26), take a first variation of this quantity and then substitute the equilibrium conditions. In the following, we note that the only requirements are $\mathbf{r}^{\prime}=0,(\mathbf{2 7}),(\mathbf{2 8})$, and Corollaries 3-5.

Note that the first variation in the first term of $\mathcal{E}$ will be identically zero, given the equilibrium condition $\mathbf{r}^{\prime}=0$. Thus, we immediately find 


$$
\delta \mathcal{E}=\frac{1}{2} \mathbf{k} \cdot\left[\delta \mathbf{A} \cdot \mathcal{J}^{-1} \cdot \mathbf{A}^{T}+\mathbf{A} \cdot \delta \mathcal{J}^{-1} \cdot \mathbf{A}^{T}+\mathbf{A} \cdot \mathcal{J}^{-1} \cdot \delta \mathbf{A}\right] \cdot \mathbf{k}-v \delta \mathcal{U}
$$

The variations $\delta \mathbf{A}, \delta \mathcal{J}^{-1}$, and $\delta \mathcal{U}$ are considered in a moment, but first we make the following observations. From Corollary 4 and 5 we know that $\mathbf{k}$ is an eigenvector of $\mathcal{J}^{-1}$ with an eigenvalue equal to $1 / \lambda$. Also, from (27) we know that $\mathbf{k}$ is an eigenvector of $\mathbf{A}$ with unit eigenvalue. Thus, we make the following simplifications:

$$
\mathcal{J}^{-1} \cdot \mathbf{A}^{T} \cdot \mathbf{k}=\mathbf{k} \cdot \mathbf{A} \cdot \mathcal{J}^{-1}=\frac{\mathbf{k}}{\lambda} \quad \text { and } \quad \mathbf{k} \cdot \mathbf{A}=\mathbf{A}^{T} \cdot \mathbf{k}=\mathbf{k}
$$

This reduces the equation to

$$
\delta \mathcal{E}=\frac{1}{2 v}\left[\frac{1}{\lambda} \mathbf{k} \cdot \delta \mathbf{A} \cdot \mathbf{k}+\mathbf{k} \cdot \delta \mathcal{J}^{-1} \cdot \mathbf{k}+\frac{1}{\lambda} \mathbf{k} \cdot \delta \mathbf{A}^{T} \cdot \mathbf{k}\right]-\delta \mathcal{U}
$$

First consider $\mathbf{k} \cdot \delta \mathbf{A} \cdot \mathbf{k}$. The general form of $\mathbf{A}$, prior to evaluating it at the equilibrium conditions, is $\mathbf{A}=\cos \phi \mathbf{U}+(1-\cos \phi) \hat{\mathbf{a}} \hat{\mathbf{a}}-\sin \phi \hat{\mathbf{a}}$. Since $\mathbf{k}$ is kept constant we can rewrite this as $\mathbf{k} \cdot \delta \mathbf{A} \cdot \mathbf{k}=\delta(\mathbf{k} \cdot \mathbf{A} \cdot \mathbf{k})$. The expression becomes $\mathbf{k} \cdot \mathbf{A} \cdot \mathbf{k}=$ $\cos \phi|\mathbf{k}|^{2}+(1-\cos \phi)(\hat{\mathbf{a}} \cdot \mathbf{k})^{2}-\sin \phi \mathbf{k} \cdot \hat{\mathbf{a}} \cdot \mathbf{k}$, and the last term is identically zero. Taking variations of this expression then gives

$$
\delta(\mathbf{k} \cdot \mathbf{A} \cdot \mathbf{k})=\sin \phi\left[(\hat{\mathbf{a}} \cdot \mathbf{k})^{2}-|\mathbf{k}|^{2}\right] \delta \phi+2(1-\cos \phi)(\hat{\mathbf{a}} \cdot \mathbf{k})(\mathbf{k} \cdot \delta \hat{\mathbf{a}}) .
$$

Now apply Corollary 4 and (27) to note that $\mathbf{a}$ and $\mathbf{k}$ are parallel to each other. This immediately zeros out the $\delta \phi$ variation term. Next, note that the variation $\delta \hat{\mathbf{a}}$ must be perpendicular to itself, and hence, must be perpendicular to $\mathbf{k}$, making the second variation term zero as well.

Now consider the term $\mathbf{k} \cdot \delta \mathcal{J}^{-1} \cdot \mathbf{k}$. First, note the identity $\delta \mathcal{J}^{-1}=-\mathcal{J}^{-1} \cdot \delta \mathcal{J} \cdot \mathcal{J}^{-1}$. This, along with Corollary 4 , tells us that $\mathbf{k} \cdot \delta \mathcal{J}^{-1} \cdot \mathbf{k}=-\mathbf{k} \cdot \delta \mathcal{J} \cdot \mathbf{k} / \lambda^{2}$. Again, since the angular momentum is a constant, this reduces to $-\delta(\mathbf{k} \cdot \mathcal{J} \cdot \mathbf{k}) / \lambda^{2}$. Consider the quantity $\mathbf{k} \cdot \mathcal{J} \cdot \mathbf{k}=\mathbf{k} \cdot \mathcal{I} \cdot \mathbf{k}-v \mathbf{k} \cdot \tilde{\mathbf{r}} \cdot \tilde{\mathbf{r}} \cdot \mathbf{k}$. The first term is identically constant and will disappear under the variation, the second term can be rewritten using the following identity, $\mathbf{k} \cdot \tilde{\mathbf{r}} \cdot \tilde{\mathbf{r}} \cdot \mathbf{k}=\mathbf{r} \cdot \tilde{\mathbf{k}} \cdot \tilde{\mathbf{k}} \cdot \mathbf{r}$. Thus, we find that

$$
\mathbf{k} \cdot \delta \mathcal{J}^{-1} \cdot \mathbf{k}=2 \frac{\mathrm{v}}{\lambda^{2}} \mathbf{r} \cdot \tilde{\mathbf{k}} \cdot \tilde{\mathbf{k}} \cdot \delta \mathbf{r} .
$$

Finally, consider the term $\delta \mathcal{U}$. Since $\mathcal{U}$ is only a function of position, when expressed in the body-fixed frame, we have the simple result $\delta \mathcal{U}=\mathcal{U}_{\mathbf{r}} \cdot \delta \mathbf{r}$, where $\mathcal{U}_{\mathbf{r}}$ signifies the gradient of $\mathcal{U}$ with respect to the position vector $\mathbf{r}$. Putting all of these elements together, we find a simplified expression for the energy variation,

$$
\delta \mathcal{E}=\left[\frac{1}{\lambda^{2}} \mathbf{r} \cdot \tilde{\mathbf{k}} \cdot \tilde{\mathbf{k}}-\mathcal{U}_{\mathbf{r}}\right] \cdot \delta \mathbf{r}
$$

Now we can apply Corollaries 4 and 5 to make the substitution $\mathbf{k} / \boldsymbol{\lambda}=\boldsymbol{\omega}$ when at the equilibrium conditions,

$$
\delta \mathcal{E}=\left[\mathbf{r} \cdot \tilde{\omega} \cdot \tilde{\omega}-\mathcal{U}_{\mathbf{r}}\right] \cdot \delta \mathbf{r} .
$$

The term multiplied by the variation $\delta \mathbf{r}$ is precisely (28), which is satisfied under the equilibrium hypothesis, and thus, we establish that $\delta \mathcal{E}=0$ to first order, that is, the energy is stationary at an equilibrium point. 
Proposition 8: A necessary and sufficient condition for a relative equilibrium is that the energy is at a stationary value and the angular momentum vector is an eigenvector of the dyad $\mathcal{J}=\mathcal{I}-v \tilde{\mathbf{r}} \cdot \tilde{\mathbf{r}}$.

That it is necessary arises from Corollary 4 and Proposition 7 . To prove sufficiency, we first need note that we can derive (40) just using the fact that $\mathbf{k}$ is an eigenvector of $\mathcal{J}$ and a unit eigenvector of $\mathbf{A}$ (the latter can be enforced trivially by choosing $\mathbf{A}=\mathbf{U}$ initially). Then, the condition that the energy is at a stationary value is reduced to a condition on the eigenvalue $\lambda$. If this is true, we can identify the angular rate $\omega$ with $\mathbf{k} / \lambda$ causing the equilibrium conditions to be identically satisfied.

\section{Locally Central Gravity Fields}

Before we consider the general case of equilibria for these equations, we first discuss a special case that has been analyzed extensively in the past, albeit in a less general way. If the relative position vector $\mathbf{r}$ and the local gravitational acceleration $\mathcal{U}_{\mathbf{r}}$ are parallel to each other, we consider this to be a "locally central" point in the gravity field. For a spherical mass distribution, we note that all points are locally central, hence, these gravity fields are generally called central gravity fields. For a symmetric mass distribution, such as an ellipsoid, we note that there are only isolated points at which the gravity field is locally central, at the six semiaxes of the body. For the ellipsoid, we note that these regions of locally central gravity coincide with the principal axes of inertia, a situation that allows for the easy identification of equilibrium configurations, which indeed most past research has focused on. For any second degree and order gravity field we find a similar result. We find, for the more general cases, that locally central points in the gravity field do not, in general, coincide with the principal axes of the inertia ellipsoid. We formalize this as follows.

Proposition 9: A locally central point in a gravity field for a SRF2BP is a relative equilibrium if and only if the position is perpendicular to a principal axis.

To show this, let

$$
\mathbf{g}=\mathcal{U}_{\mathbf{r}}
$$

Thus, at a locally central point we have $\mathbf{g}=-g \hat{\mathbf{r}}$ (we note that the vectors are antiparallel in general). Now, we can rewrite (28) and (29) and evaluate Proposition 1 to find

$$
\begin{gathered}
\left(g-\omega^{2} r\right) \hat{\mathbf{r}}=0 \\
\boldsymbol{\omega} \times \mathcal{I} \cdot \boldsymbol{\omega}=0 \\
\boldsymbol{\omega} \cdot \hat{\mathbf{r}}=0 .
\end{gathered}
$$

All of these equations, taken together, place constraints on the angular velocity vector. From (44) we see that $\boldsymbol{\omega}$ must lie along a principal axis of the inertia ellipsoid. From (45) we see that $\boldsymbol{\omega}$ must also be perpendicular to the position vector. Equation (43) just provides us with the necessary magnitude of the rotation rate once the other equations are satisfied. Then, if the position vector is not perpendicular to a principal axis (44) cannot be satisfied, because $\omega \neq 0$ in general and the gravity (and position vector in this case) must be perpendicular to the spin axis from Proposition 1. The necessary conditions follow directly. 
We see that for ellipsoids, where the locally central points are aligned with the principal axes, there are four distinct rotation directions that are equilibria, two associated with each of the other principal axes. For a general mass distribution, however, we find that this situation is not true. In fact, for any body with non-zero coefficients in their odd degrees and/or orders in their harmonic gravity fields this situation will not occur generically.

\section{Reduction of the Equilibrium Conditions for Non-Locally Central Points}

To determine an equilibrium condition, we must solve six equations, (28) and (29), for six unknowns $\mathbf{r}$ and $\boldsymbol{\omega}$. We note, however, that we expect there to be fourfold degeneracies in these solutions due to the existence of 4 integrals of motion, stated in (31) and (32). Thus, we expect to be able to reduce these six conditions to just two independent conditions for equilibrium. Formally, we could perform these reductions directly from the integrals, but this is somewhat tedious and does not take advantage of certain symmetries that are present in the physical problem we are considering. In the following, we make the tacit assumption that $\mathbf{r}$ and $\mathbf{g}$ are not collinear.

The equilibrium condition we find below provides two conditions for the three components of relative position $\mathbf{r}$. In practice, we can specify one element of the position vector as a free parameter, and solve for the two other components as functions of this. A natural parameter to choose for the free parameter is the radius of the position vector, since we expect solutions to exist at all values of radius. We represent these equilibrium conditions as a function of $\hat{\mathbf{r}}$, which can also be represented as the two angles that define the relative position in the body-fixed frame. Then, these two equations are solved for the angular location of the relative position vector for a given value of radius. For each value of radius there are multiple solutions.

In our work we have also found various forms of the equilibrium conditions that only provide necessary or sufficient conditions for an equilibrium. These will be described in more detail in a future paper, but are not discussed for the current analysis. In the following we only provide a necessary and sufficient condition for an equilibrium to exist. Although this approach is used in the following to find some specific examples of relative equilibria, it suffers from some disadvantages as an algorithm that cause us to not use it to completely replace the two conditions briefly mentioned above. Specifically, we have found poor convergence in solving for relative equilibria where the system spins in the vicinity of a non-maximum moment of inertia.

At the heart of this approach is Proposition 8. Given a trial value for $\mathbf{r}$ we can form the dyad $\mathcal{J}$ and delineate the three mutually orthogonal eigenvectors and their eigenvalues, denoted by $\mathbf{v}_{i}$ and $\lambda_{i}, i=1,2,3$. Choose one of these eigenvectors to be aligned with the system angular momentum (we can choose this freely since the angular momentum is a free parameter of the system) and assume that the axis of rotation is initially aligned in this direction as well, denote this eigenvector and eigenvalue by $\mathbf{v}$ and $\lambda$. Given this, the remaining condition for an equilibrium is

$$
\frac{1}{\lambda^{2}} \mathbf{r} \cdot \tilde{\mathbf{k}} \cdot \tilde{\mathbf{k}}-U_{\mathbf{r}}=0
$$


If we assume a value for $|\mathbf{k}|$ we then have three equations for three unknowns, the elements of the position vector $\mathbf{r}$. Again, we find it convenient to decompose this equation into three transverse directions. We consider the projection of this equation into the three directions $\mathbf{r}, \mathbf{v}$, and $\mathbf{v} \times \mathbf{g}$, where $\mathbf{g}=\mathcal{U}_{\mathbf{r}}$.

Projecting (46) along $\mathbf{r}$ we find

$$
\frac{k^{2}}{\lambda^{2}} \mathbf{r} \cdot \tilde{\mathbf{v}} \cdot \tilde{\mathbf{v}} \cdot \mathbf{r}-\mathbf{r} \cdot \mathbf{g}=0
$$

We can solve this equation for the magnitude of the angular momentum,

$$
k^{2}=-\lambda^{2} \frac{\mathbf{r} \cdot \mathbf{g}}{r^{2}\left[1-(\hat{\mathbf{r}} \cdot \hat{\mathbf{v}})^{2}\right]} .
$$

Instead of specifying the angular momentum magnitude, we can use this equation with a set value of the radius to define the angular momentum magnitude. In this way, we can easily eliminate one of the unknowns.

Proposition 9: The following two conditions are necessary and sufficient for a relative equilibrium to exist:

$$
\begin{gathered}
\mathbf{v} \cdot \mathbf{g}=0 \\
\mathbf{v} \cdot \tilde{\mathbf{r}} \cdot \mathbf{g}=0 .
\end{gathered}
$$

To establish this, project (46) along $\mathbf{v}$. The first term is identically zero, because $\mathbf{v} \cdot \tilde{\mathbf{v}}=0$. The remaining condition is $\mathbf{v} \cdot \mathbf{g}=0$. Next, project along $\mathbf{v} \times \mathbf{g}$, which is orthogonal to $\mathbf{g}$, to find $\mathbf{v} \cdot \tilde{\mathbf{g}} \cdot \mathbf{r}=\mathbf{v} \cdot \tilde{\mathbf{r}} \cdot \mathbf{g}=0$. These two conditions constitute the necessary and sufficient conditions for equilibrium.

In practice, we keep $r$ constant and only vary $\hat{\mathbf{r}}$ to satisfy the two conditions. This method has the advantage of letting us control the placement of the angular velocity. A drawback of the sufficiency conditions derived previously is that there is no direct control over which possible eigenvector orientation the solution is drawn to.

\section{SOLUTION OF THE EQUATIONS}

Now we consider the solution of the equilibrium conditions for general gravity fields and inertia tensor. For a general gravity field we cannot hope to find analytic solutions to these equations, although it may be possible to develop local solutions under the assumption that $r \gg 1$ by expanding the gravity field to the lowest order. We do not pursue such an approach here, but instead describe how we can solve these equations under general conditions when the two bodies are in close proximity to each other. To motivate our general approach, we will use a realistic asteroid shape model for Toutatis, and its associated constant density gravity field. ${ }^{8}$ We use this asteroid due to its clearly non-symmetric shape. ${ }^{9}$ (See FIGURES 1-7.)

We have investigated solutions to the sufficient and necessary and sufficient conditions. We have found, for reasons not fully understood, that the sufficient conditions are better suited to solving for hyperbolically unstable relative equilibria, whereas the necessary and sufficient condition is better suited to finding the stable relative equilibria and the complex unstable relative equilibria. We suspect this difference is due to the saddle structure of the force potential for one versus the local 


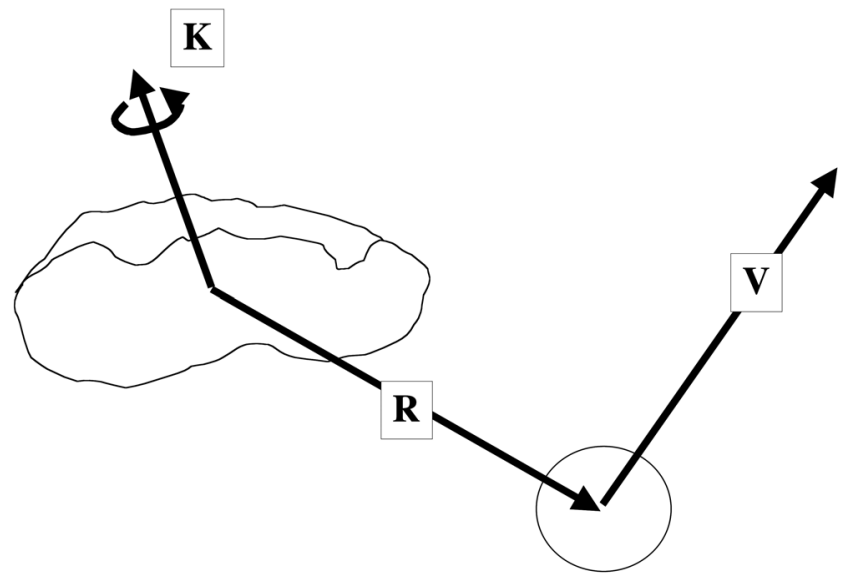

FIGURE 1. Representation of a sphere-restricted full two-body problem.

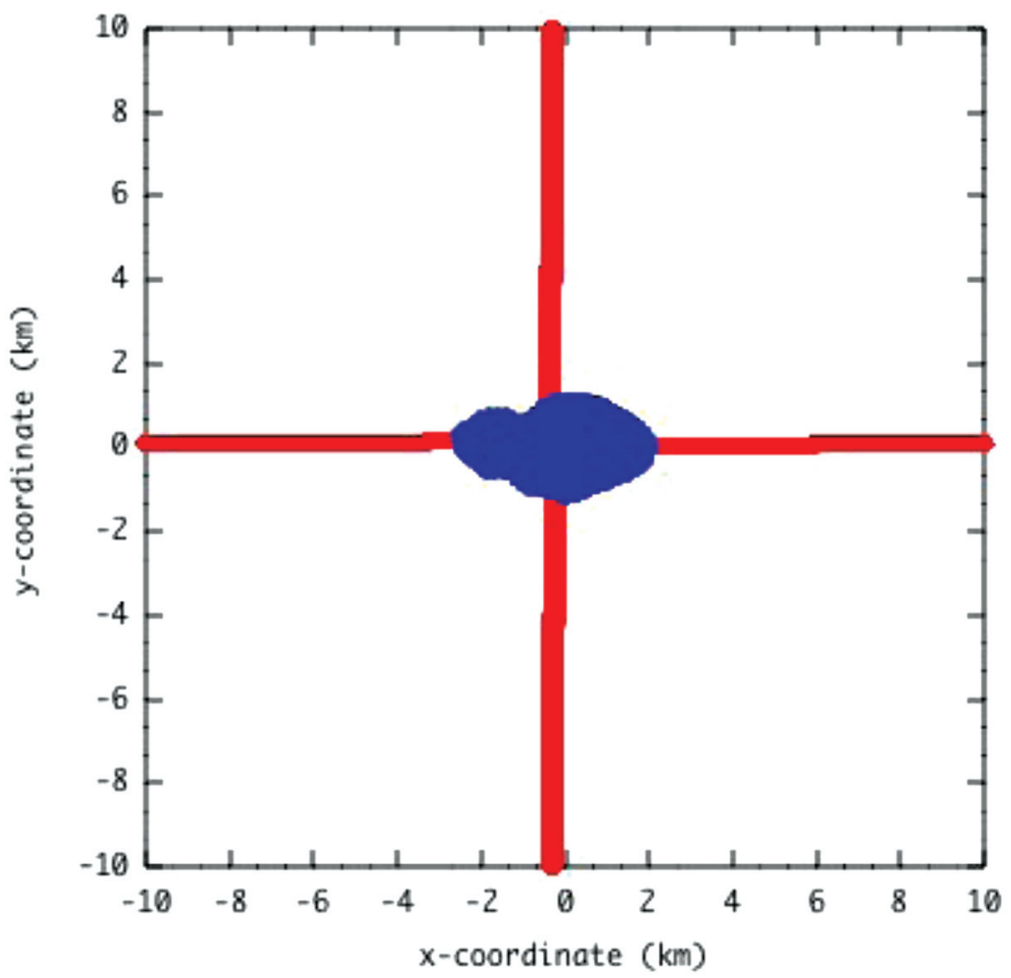

FIGURE 2. Locus of relative equilibria about maximum moment of inertia for Toutatis. 


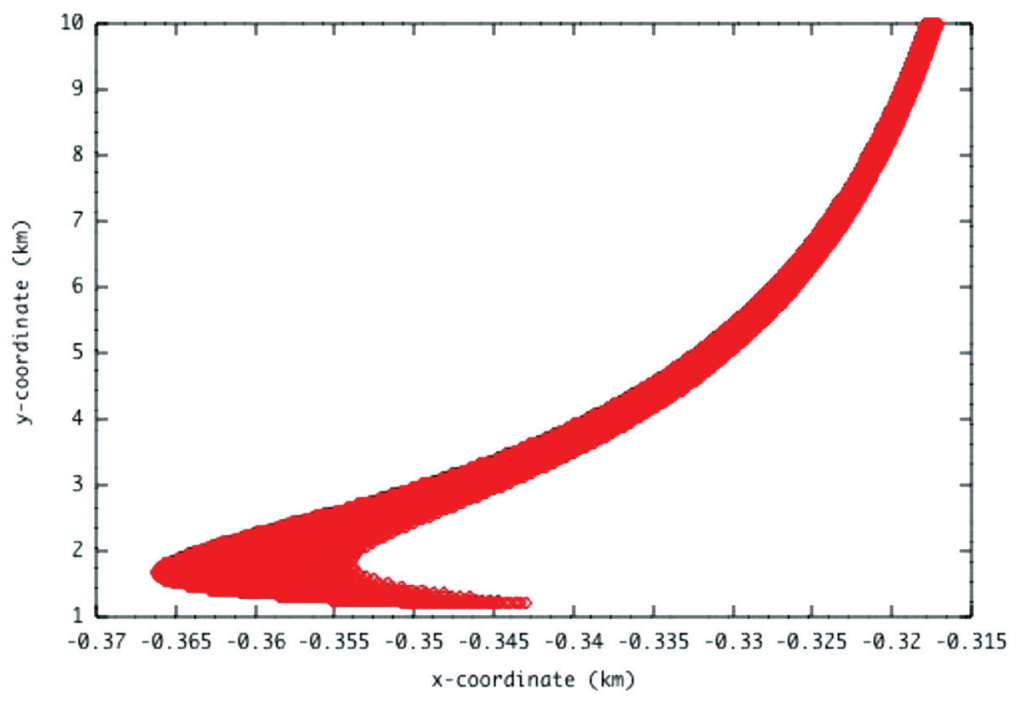

FIGURE 3. Detail of location of relative equilibria.

maximum/minimum structure of the other. In particular, we found that the sufficient condition could not find stable equilibria, but would only converge on equilibria with a saddle structure. The necessary and sufficient energy minimizer solution, on the other hand, could easily converge on the stable equilibrium. We note that it could not converge on the saddle equilibria, however. We hypothesize that the local structure of the equilibrium point controls this aspect of solution.

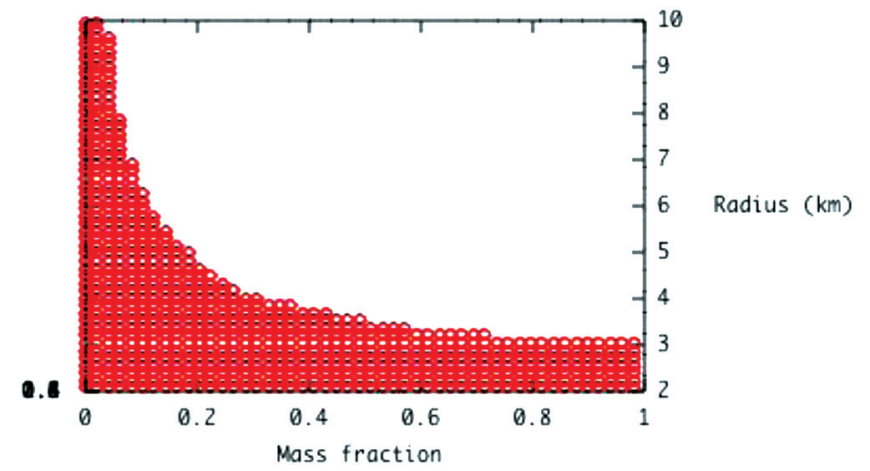

FIGURE 4. Stability of relative equilibria on the $+x$ axis: $\diamond$, unstable relative equilibria. 


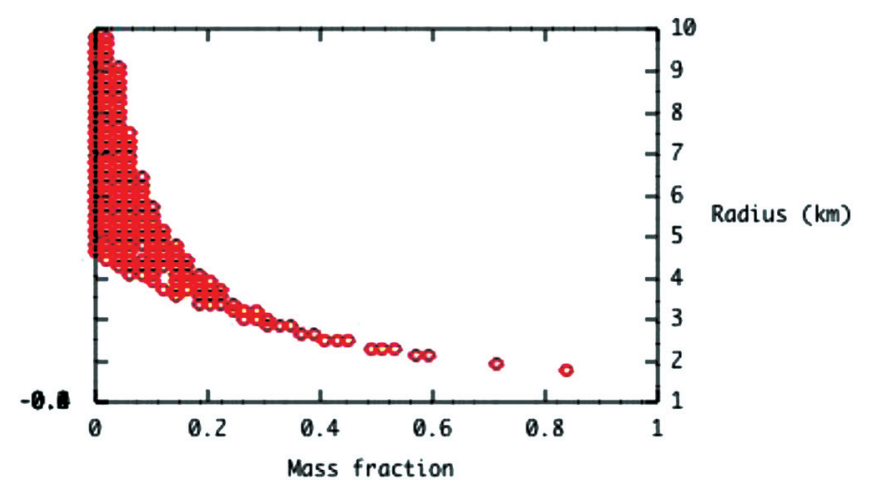

FIGURE 5. Stability of relative equilibria on the $-y$ axis: $\diamond$, stable relative equilibria.

We present results for relative equilibria at a range of distances and mass parameters $v$ for Toutatis. All these results assume rotation about the maximum moment of inertia of the system, and the use of the necessary and sufficient conditions from Proposition 11 to compute the relative equilibrium. We note that these results are qualitatively similar to those found for an ellipsoid, ${ }^{5}$ albeit with obvious deviations due to the asymmetry of the mass distribution.

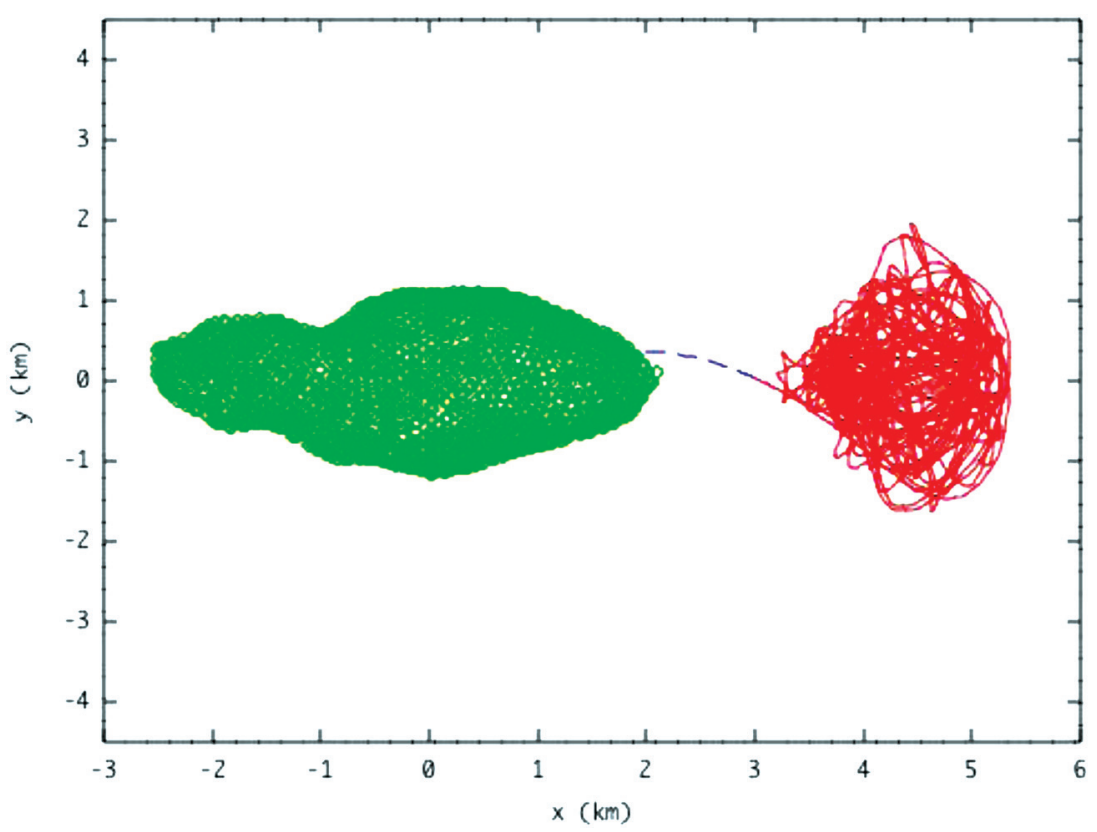

FIGURE 6. Unstable, Hill stable trajectory in $x-y$ plane, $v=0.5, r=3$. 


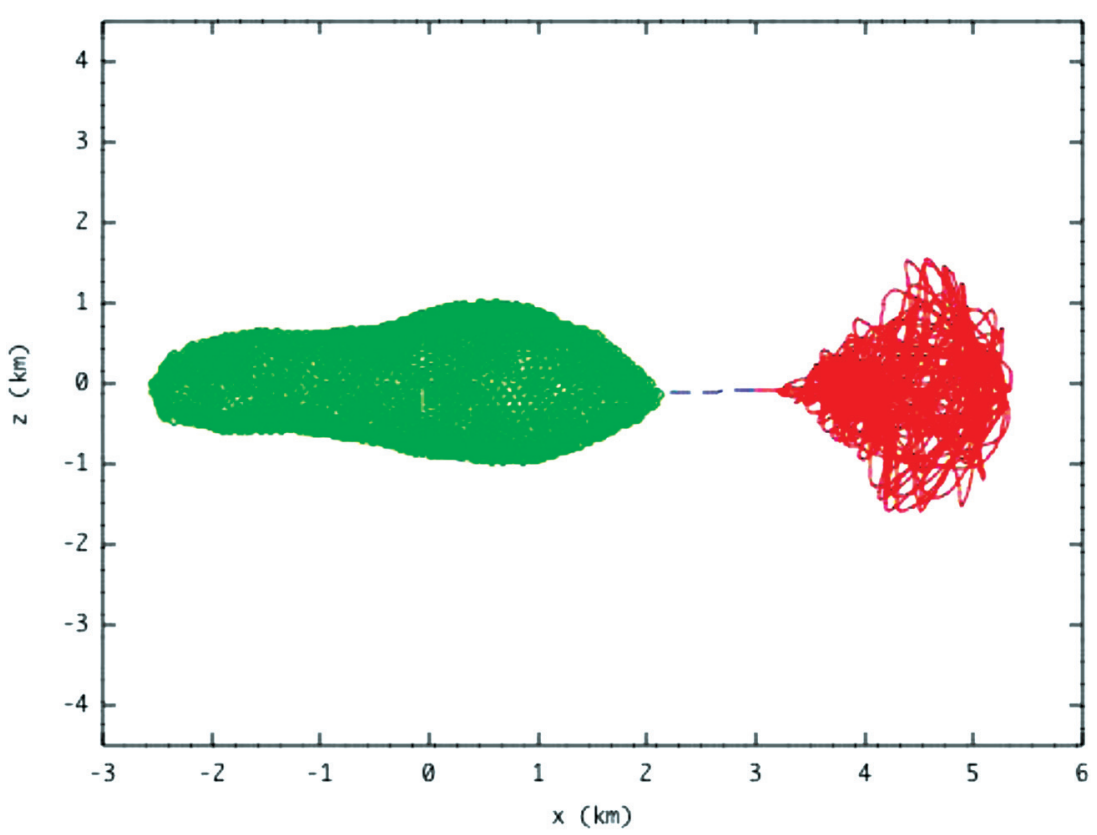

FIGURE 7. Unstable, Hill stable trajectory in $x-z$ plane, $v=0.5, r=3$.

\section{ACKNOWLEDGMENTS}

This work was supported by grants to the University of Michigan from the NASA Planetary Geology and Geophysics program and by the Jet Propulsion Laboratory/ California Institute of Technology.

\section{REFERENCES}

1. Maciejewski, A.J. 1995. Reduction, relative equilibria and potential in the two rigid bodies problem. Celest. Mech. Dyn. Astron. 63: 1-28.

2. WANG, L.-I., P.S. KRIShNAPRASAD \& J.H. MadDOCKS. 1991. Hamiltonian dynamics of a rigid body in a central gravitational field. Celest. Mech. Dynam. Astron. 50: 349386.

3. Simo, J.C., D. Lewis \& J.E. Marsden. 1991. Stability of relative equilibria. I. The reduced energy-momentum method. Arch. Rational Mech. Anal. 115(1): 15-59.

4. Scheeres, D.J. 2002. Stability in the full two-body problem. Celest. Mech. Dynam. Astron. 83: 155-169.

5. SCHEERES, D.J. 2004. Stability of relative equilibria in the full two-body problem. In Astrodynamics, Space Missions, and Chaos. E. Belbruno, D. Folta \& P. Gurfil, Eds. Ann. N.Y. Acad. Sci. 1017: 81-94.

6. Koon, W.-S., J.E. Marsden, S. Ross, et al. 2004. Geometric mechanics and the dynamics of asteroid pairs. In Astrodynamics, Space Missions, and Chaos. E. Belbruno, D. Folta \& P. Gurfil, Eds. Ann. N.Y. Acad. Sci. 1017: 11-38.

7. Scheeres, D.J., S.J. Ostro, R.S. Hudson \& R.A. Werner. 1996. Orbits close to asteroid 4769 Castalia. Icarus 121: 67-87. 
8. Werner, R.A. \& D.J. ScheERES. 1997. Exterior gravitation of a polyhedron derived and compared with harmonic and mascon gravitation representations of asteroid 4769 Castalia. Celest. Mech. Dynam. Astron. 65: 31344.

9. Scheeres, D.J., S.J. Ostro, R.S. Hudson, et al. 1998. Dynamics of orbits close to asteroid 4179 Toutatis. Icarus 132: 53-79. 